\title{
Social Anxiety and Obsessive-Compulsive Disorder Are Common Among Persons With Multiple Sclerosis at King Abdulaziz Medical City, Riyadh
}

Ismail A. Khatri ${ }^{1,2,3}$, Sarah Aljwair ${ }^{1}$, Hajar Alammar ${ }^{1}$, Amjad Altariq ${ }^{1}$, Nazish Masud ${ }^{4}$, Yaser Al Malik ${ }^{1}$ , Suleiman Kojan ${ }^{5}$

1. Neurology, King Saud Bin Abdulaziz University for Health Sciences College of Medicine, Riyadh, SAU 2. Neurology, King Abdullah International Medical Research Center, Riyadh, SAU 3. Division of Neurology, Department of Medicine, King Abdulaziz Medical City, Ministry of National Guard Health Affairs, Riyadh, SAU 4. Medical Education, King Saud Bin Abdulaziz University for Health Sciences, Riyadh, SAU 5. Neurology, Oakland University William Beaumont School of Medicine, Royal Oak, USA

Corresponding author: Ismail A. Khatri, ismailkhatri@yahoo.com

\section{Abstract}

\section{Background}

Multiple sclerosis (MS) is associated with a physical disability and disturbed psychosocial functioning in young people. Many psychological and psychiatric comorbidities have been reported in MS.

\section{Objective}

To determine the frequency of social anxiety disorder (SAD) and obsessive-compulsive disorder (OCD) among MS patients and their relation to MS severity.

\section{Methods}

A cross-sectional survey was conducted in an adult MS cohort. Yale-Brown Obsessive-Compulsive Scale (YBOCS) and Social Phobia Inventory (SPIN) were used to determine the presence and severity of OCD and SAD. The Statistical Package for the Social Sciences (SPSS) version 22 (IBM Corp., Armonk, NY) was used for statistical analysis. The Mann-Whitney $\mathrm{U}$ test and logistic regression were used to assess the association of the two diseases with the severity of MS.

\section{Results}

A total of 145 persons with MS (pwMS) were studied. The mean age was $33.5( \pm 8.5)$ years; the mean duration of MS was $7.2( \pm 5.1)$ years. The majority $(74.1 \%)$ were women; $57.3 \%$ were married; $63 \%$ had a college education; $50 \%$ belonged to the higher middle-class socioeconomic strata. Relapsing-remitting multiple sclerosis was the most common type of MS (92.2\%). The mean Expanded Disability Status Scale (EDSS) score was $2.24( \pm 2.19)$. SAD was reported by $26.9 \%$, and OCD was reported by $31 \%$ of the cohort. PwMS with walking difficulty but not wheelchair-bound had a statistically significant increased risk of SAD $(p=$ 0.036). There was no direct association between MS-related disability and OCD. However, pwMS with SAD were more likely to have concomitant OCD ( $t=4.68$, $p$-value $<0.001$, 95\% CI: $0.47-1.16)$. Increasing disability was associated with higher chances of developing social anxiety and, in turn, $\mathrm{OCD}(\mathrm{t}=3.39$, $\mathrm{p}$-value $<0.001$, 95\% CI: 0.66-2.52).

\section{Conclusions}

Social anxiety and obsessive-compulsive disorders were present in nearly one-third of pwMS. Impaired walking but not wheelchair dependence was associated with social anxiety. PwMS with SAD were more likely to have obsessive-compulsive disorder.

Categories: Neurology

Keywords: multiple sclerosis, social anxiety disorder, obsessive compulsive disorder, saudi arabia, disability, psychosocial function

\section{Introduction}

Multiple sclerosis (MS) is a neurodegenerative, progressive, multifocal demyelinating disease of the central nervous system [1]. The demyelination is caused by an immune-mediated inflammation likely resulting from an interaction between genetic and environmental factors [2]. The most common pattern of MS is the relapsing-remitting MS (RRMS) with a prevalence of ( $85 \%-90 \%)$, followed by secondary progressive MS 
(SPMS) and primary progressive MS (PPMS) [3]. The global prevalence of MS is 30 per 100,000, with an incidence rate of 2.5 per 100,000 per year [4]. Women constitute the majority of pwMS with a 2:1 ratio [4]. MS in the Middle East North Africa region has increased consistently with the growing global prevalence of the disease [5]. In a recent Saudi study, the prevalence of MS was 40.4/100,000 population, with threefourths of pwMS younger than 40 years of age and a female to male ratio of 2:1 [6]. A local Saudi study from 1998 suggested that the onset was mostly in the third decade, with the majority of pwMS having RRMS [7].

A systemic review, investigating the prevalence of psychological disorders and their comorbidity in pwMS showed that the most common psychological comorbidities were depression and anxiety [8]. A Saudi study of 24 patients found that depression (20.8\%) was more common than anxiety (4.2\%) [9]. Prior studies have shown a high frequency of social anxiety disorder (SAD) and obsessive-compulsive disorder (OCD) among pwMS [10-11]. SAD is characterized by the fear of negative evaluation from others, somatic symptoms such as palpitation and sweating, social isolation, and avoidance behavior [10]. Epidemiological studies have shown the prevalence of SAD in the global population to be $2 \%-16 \%$ [12]. In a study of 251 pwMS, $30.6 \%$ had social anxiety symptoms [10]. OCD is characterized by a combination of obsessions such as repetitive thoughts and compulsions, i.e. repetitive behaviors [13]. Both are attributed to or exacerbated by anxiety. In the general population, OCD is estimated to have a $2 \%$ lifetime prevalence [12]. In a study of pwMS, OCD was found in $16.1 \%$ of pwMS that correlated with a higher EDSS score [11]. Previous studies evaluated the frequencies of SAD and OCD in pwMS in different cultural backgrounds [10-11]. One of the studies was performed in Canada, and the other was in the Iranian population. Both studies showed a significant burden of these conditions despite different cultural backgrounds.

SAD and OCD are among major causes of functional impairment worldwide [14-15]. They lead to social isolation and are associated with a low quality of life. Social anxiety is associated with occupational instability, poor economical and marital status [14]. OCD accounts for significant emotional and functional disability and severe family burden [15]. In pwMS, studying any aspect of the disease, including emotional and social aspects, which may affect the course of the disease or alter the disability is highly significant and may alter the management plan. There is very limited data on psychiatric comorbidities in pwMS from the region. Our study aimed to determine the frequency of social anxiety disorder and OCD among pwMS in a tertiary care center and to examine their association with the disability status of the patients.

This study was earlier presented as a poster at the 19th Department of Medicine Research Day at King Abdulaziz Medical City, Ministry of National Guard - Health Affairs (MNGHA), Riyadh, Saudi Arabia, on November 22, 2018.

\section{Materials And Methods}

Ethical approval for the study was obtained from the institutional review board (IRB) of King Abdullah International Medical Research Center (KAIMRC) and informed consent was obtained from persons with MS. A cross-sectional survey was conducted among pwMS visiting the outpatient neurology clinic between October 2017 and August 2018 at King Abdulaziz Medical City, MNGHA, Riyadh, Kingdom of Saudi Arabia. All adult pwMS, 16 years and above, who consented to participate were included. Multiple sclerosis was classified according to the clinical phenotype [16]. Those who did not have a conclusive diagnosis or had a cognitive disability were excluded from the study. A sample size of 139 was estimated with a margin of error of $5 \%$, a confidence level of $95 \%$, and a response distribution of $30.6 \%$ using the Raosoft sampling calculator (Raosoft, Inc., Seattle, WA) [10]. The non-probability convenience sampling technique was used for the inclusion of pwMS, as the data was collected in the hospital setting and pwMS were contacted based on availability at the time of data collection. A total of 145 pwMS were included in the study.

The Social Phobia Inventory (SPIN) and Yale-Brown Obsessive Compulsive Scale (Y-BOCS), both validated tools, were used to identify the presence of social anxiety and OCD symptoms [17-18]. The questionnaires were translated into Arabic by native Arabic speakers [19], and all the steps of translation, including forward and backward translation, were done followed by a pilot testing with 108 participants other than the ones included in the data analysis. The sample size of 108 for pilot testing was estimated based on the total number of items, which was 27 , as recommended for reliability testing. Four pwMS per item were considered. Based on the pilot testing, item numbers 3, 6, 7, 8, 13, 14, and 15 of the SPIN questionnaire and item numbers $1,2,7$, and 8 of the YOBCS questionnaire were reworded and the final questionnaire in Arabic was drafted. The Cronbach for the Y-BOCS 10 items was 0.90 while for the 17 items of the SPIN questionnaire, it was 0.91 . The over-reliability score for the total 27 items of both questionnaires was 0.93 , showing good internal reliability of the translated version of the questionnaires in our sample. The expanded disability status scale (EDSS) score was obtained from the treating neurologist's assessment and was used for quantifying the disability in pwMS. Some patients suffered limitations, including visual impairment, motor disability, and illiteracy. For such pwMS, the questionnaire was filled by the co-investigators after asking the inventory questions in simple language and explaining the choice of answer.

The data were analyzed using the Statistical Package for Social Sciences (SPSS) version 22 (IBM Corp., Armonk, NY). The descriptive analysis was performed and the numerical variables were presented as mean and standard deviation, whereas the categorical data were presented as percentages and proportions. Initially, the total scores for SPIN and Y-BOCS were computed by a summation of all the items for each 


\section{Cureus}

\begin{tabular}{|c|c|c|c|}
\hline \multicolumn{2}{|l|}{ Variables } & Frequency & Percentage $\%$ \\
\hline \multicolumn{2}{|l|}{ Age (Mean $\pm S D)$} & $33.48 \pm 8.54$ & \\
\hline \multicolumn{2}{|l|}{ Duration of MS $\pm($ Mean $\pm S D)$} & $7.16 \pm 5.15$ & \\
\hline \multirow{3}{*}{ Gender } & Female & 106 & 73.1 \\
\hline & Male & 37 & 25.5 \\
\hline & Missing information & 2 & 1.4 \\
\hline \multirow{3}{*}{ Marital status } & Single & 56 & 39.2 \\
\hline & Married & 82 & 57.3 \\
\hline & Divorced & 5 & 3.5 \\
\hline \multirow{4}{*}{ Monthly family income } & $<10,000$ riyals & 38 & 27.9 \\
\hline & $11,000-20,000$ riyals & 49 & 36.0 \\
\hline & $>20,000$ riyals & 19 & 14.0 \\
\hline & Not known & 29 & 21.3 \\
\hline \multirow{8}{*}{ Education } & No education & 4 & 2.8 \\
\hline & Elementary & 5 & 3.4 \\
\hline & Middle & 3 & 2.1 \\
\hline & High school & 37 & 25.5 \\
\hline & Bachelor & 85 & 58.6 \\
\hline & Master & 6 & 4.1 \\
\hline & Doctor/ PhD & 1 & 0.7 \\
\hline & Other & 4 & 2.8 \\
\hline \multirow{6}{*}{ Type of MS } & Relapsing Remitting MS & 118 & 92.2 \\
\hline & Primary Progressive MS & 3 & 2.3 \\
\hline & Secondary Progressive MS & 5 & 3.9 \\
\hline & Unknown course & 2 & 1.6 \\
\hline & No medication & 20 & 14.1 \\
\hline & Interferon beta 1a (Avonex) & 18 & 12.7 \\
\hline
\end{tabular}




\section{Cureus}

\begin{tabular}{|c|c|c|c|}
\hline \multirow{9}{*}{ Medication } & Interferon beta 1a (Rebif) & 20 & 14.1 \\
\hline & Interferon beta $1 \mathrm{~b}$ (Betaferon) & 3 & 2.1 \\
\hline & Fingolimod (Gilenya) & 32 & 22.5 \\
\hline & Teriflunomide (Aubagio) & 8 & 5.6 \\
\hline & Dimethyl fumarate (Tecfidera) & 2 & 1.4 \\
\hline & Natalizumab (Tysabri) & 31 & 21.8 \\
\hline & Alemtuzumab (Lemtrada) & 5 & 3.5 \\
\hline & Ocrelizumab (Ocrevus) & 1 & 0.7 \\
\hline & Rituximab & 2 & 1.4 \\
\hline
\end{tabular}

TABLE 1: Demographic profile, type of multiple sclerosis, and current use of medications ( $n=$ 145)

Social anxiety disorder was reported by 39 (26.9\%) of the participants, and OCD was reported by 45 (31\%) of the participants. Most of the pwMS with SAD were categorized as mild 20 (51\%), followed by moderate 13 (33\%), and severe 5 (13\%), while only one (3\%) patient had extremely severe SAD. Among those who had OCD, mild symptoms 34 (76\%) were the most prevalent, followed by moderate $9(20 \%)$ and $2(4 \%)$ had severe OCD, while none had extreme OCD. The EDSS score was available for 138 pwMS with a mean of $2.24 \pm 2.19$. Based on EDSS, 106 (77\%) patients were fully ambulatory, 10 (7\%) had some walking difficulty, and 2 (16\%) were restricted to wheelchairs. The responses to various questions of SPIN and Y-BOCS are shown in Table 2. Disease severity and the proportions of SAD and OCD are presented in Table 3. 


\section{Cureus}

Descriptive statistics of SPIN and Y-BOCS

1. I am afraid of people in authority

2.1 am bothered by blushing in front of people

3. Parties and social events scare me

4. I avoid talking to people I don't know

5. Being criticized scares me a lot

6. Fear of embarrassment causes me to avoid doing things or speaking to people

7. Sweating in front of people causes me distress

8. I avoid going to parties

9. I avoid activities in which I am the center of attention

10. Talking to strangers scares me

11. I avoid having to give speeches

13. Heart palpitations bother me when I am around people

14. I am afraid of doing things when people might be watching

15. Being embarrassed or looking stupid is among my worst fears

16. I avoid speaking to anyone in authority

17. Trembling or shaking in front of others is distressing to me
12. I would do anything to avoid being criticized

\begin{tabular}{|c|c|c|c|c|}
\hline Not at all & A little bit & Somewhat & Very much & Extremely \\
\hline N (\%) & N (\%) & N (\%) & N (\%) & N (\%) \\
\hline $91(64.1)$ & 27 (19) & 15 (10.6) & 7 (4.9) & 2 (1.4) \\
\hline 84 (58.3) & 38 (26.4) & 15 (10.4) & $4(2.8)$ & $3(2.1)$ \\
\hline 76 (52.8) & $29(20.1)$ & $20(13.9)$ & $12(8.3)$ & $7(4.9)$ \\
\hline $80(55.2)$ & $34(23.4)$ & 18 (12.4) & $9(6.2)$ & $4(2.8)$ \\
\hline $58(40)$ & 48 (33.1) & $15(10.3)$ & $16(11)$ & $8(5.5)$ \\
\hline 75 (52.8) & 35 (24.6) & $20(14.1)$ & $6(4.2)$ & $6(4.2)$ \\
\hline $96(66.2)$ & $24(16.6)$ & $13(9)$ & $9(6.2)$ & $3(2.1)$ \\
\hline 77 (53.1) & $29(20)$ & 20 (13.8) & $13(9)$ & $6(4.1)$ \\
\hline 67 (46.2) & $4(29)$ & $15(10.3)$ & $19(13.1)$ & $2(1.4)$ \\
\hline $102(71.3)$ & 24 (16.8) & $13(9.1)$ & $2(1.4)$ & $2(1.4)$ \\
\hline $\begin{array}{l}69 \\
(47.6 \%)\end{array}$ & 32 (22.1) & $19(13.1)$ & $17(11.7)$ & $8(5.5)$ \\
\hline $\begin{array}{l}71 \\
(49.3 \%)\end{array}$ & 32 (22.2) & 23 (16) & $12(8.3)$ & $6(4.2)$ \\
\hline $\begin{array}{l}80 \\
(55.9 \%)\end{array}$ & $33(23.1)$ & 20 (14) & $6(4.2)$ & $4(2.8)$ \\
\hline $\begin{array}{l}64 \\
(44.4 \%)\end{array}$ & $36(25)$ & 20 (13.9) & $18(12.5)$ & $6(4.2)$ \\
\hline $\begin{array}{l}60 \\
(41.4 \%)\end{array}$ & $43(29.7)$ & $16(11)$ & 21 (14.5) & 5 (3.4) \\
\hline $\begin{array}{l}89 \\
(61.8 \%)\end{array}$ & 31 (21.5) & $13(9)$ & $7(4.9)$ & $4(2.8)$ \\
\hline $\begin{array}{l}67 \\
(46.5 \%)\end{array}$ & $35(24.3)$ & $21(14.6)$ & $10(6.9)$ & $11(7.6)$ \\
\hline
\end{tabular}

TABLE 2: Descriptive statistics of SPIN $(1-17)$ and Y-BOCS $(18$ - 27) questions 


\section{Cureus}

\begin{tabular}{|c|c|c|}
\hline & & $\mathrm{n}(\%)$ \\
\hline \multirow{3}{*}{ EDSS categories $\mathrm{n}=138$} & Fully ambulatory & $106(77)$ \\
\hline & Some walking difficulty & $10(7)$ \\
\hline & Restricted to wheelchair & $22(16)$ \\
\hline \multirow{4}{*}{ Severity of SAD based on SPIN score $(n=39)$} & Mild & $20(51)$ \\
\hline & Moderate & $13(33)$ \\
\hline & Severe & $5(13)$ \\
\hline & Very Severe & 1 (3) \\
\hline \multirow{3}{*}{ Severity of OCD based on YOBCS score $(n=45)$} & Mild case & $34(76)$ \\
\hline & Moderate case & $9(20)$ \\
\hline & evere cas & $2(4)$ \\
\hline
\end{tabular}

TABLE 3: Frequency of social anxiety disorder, obsessive-compulsive disorder, and EDSS score among persons with MS

EDSS: Expanded Disability Status Scale; SPIN: Social Phobia Inventory; Y-BOCS: Yale-Brown Obsessive Compulsive Scale

Fisher's exact test revealed a significant association between the EDSS scores, suggesting walking difficulty and clinically significant symptoms of social anxiety measured by SPIN with a p-value of 0.036 . Social anxiety was more likely to be present in those pwMS who were not wheelchair-bound but walking with difficulty. There was a significant association of clinically significant SPIN (score 19 ) with Y-BOCS using the two-tailed Mann-Whitney U test ( $\mathrm{U}=1404$; p-value $<0.001$ ). The Y-BOCS score of 145 pwMS had a mean of $6 \pm 6.02$.

Multiple linear regression modeling showed Y-BOCS scores and EDSS together as a significant predictor of social anxiety (SPIN) with ( $\mathrm{t}=4.68$, p-value $<0.001,95 \% \mathrm{CI}: 0.47-1.16)$ and $(\mathrm{t}=3.39$, p-value $<0.001,95 \% \mathrm{CI}$ : 0.66-2.52), respectively. Age and duration of MS were removed from the modeling based on the low Rsquared value. The result of linear regression modeling suggested that pwMS who had social anxiety were more likely to have concomitant OCD and the higher the disability (EDSS scores), the higher the chance of social anxiety.

\section{Discussion}

Social anxiety and OCD were frequent in our persons with multiple sclerosis. SAD was found in 39 (26.9\%), which was significantly higher than the $2 \%-16 \%$ frequency in the general population [12]. Studies have reported the prevalence of social anxiety $19 \%$ to $30 \%$ among pwMS [10,21]. A systematic review found the prevalence of social anxiety in pwMS to be $21.9 \%$ [8]. Our findings are consistent with a previously reported frequency of SAD in pwMS. The relative differences in the results can be partially attributed to the different scales used in the assessment of social anxiety. Interestingly, we found this relation most significant in those who were still ambulatory but had difficulty in walking. This has not previously been reported in the literature. In the study from Canada, although approximately 30\% of pwMS reported anxiety, there was no correlation to neurological disability [10]. We believe that this finding can be the result of insecurity or fear of embarrassment of falling in public. We think this was due to the apprehension those pwMS felt while walking. Those who become wheelchair-bound probably accepted their disability more. Additionally, wheelchair-bound pwMS are probably less likely to attend social gatherings as compared to those who are still walking. One of the items in SPIN inquiring about anxiety related to trembling and shaking in public was reported to be extremely distressing by 11 (7.6\%) and very much distressing by 10 (6.9\%), again suggesting a possible feeling of embarrassment due to disability. Other questions that got the highest scores in SPIN were fear of being embarrassed, looking stupid, or being criticized, which are common features of social anxiety $[10]$.

There are not many studies investigating the prevalence of OCD in Saudi Arabia in general and multiple sclerosis in particular. We found that 45 (31\%) of the pwMS were suffering from OCD, which is much higher than the prevalence of OCD in the general population 2\% [12]. Our findings were comparable with other studies that showed similar results in pwMS [22-24]. In one study, the prevalence of OCD among pwMS was somewhat lower at $16.1 \%$ [11]. These dissimilarities might result from the differences in sample size, cultural differences, time constraints, and educational levels among pwMS. In our study, obsessive thoughts were 
Most of our pwMS had mild to moderate disability based on EDSS, hence the results may not be representative for pwMS with a severe disability. This could be attributed to the fact that most of the data were collected from outpatient clinics. Additionally, persons with severe MS are more likely to have cognitive dysfunction and were excluded from our study.

Our results also showed a significant relationship between elevated EDSS scores and social anxiety symptoms. Our results showed that pwMS with high EDSS scores were more likely to have SAD, and the pwMS who had SAD were, in turn, more likely to have OCD. Thus, the higher the EDSS scores the higher the chances of getting SAD as well as OCD. It was uncertain whether SAD developed first or OCD developed first. Social anxiety was probably more likely to develop first, as we found an independent relationship of SPIN scores to EDSS scores. Baldwin et al., exploring the relationship between OCD and SAD, thought that SAD was more likely to be secondary in pwMS with a primary OCD diagnosis [25].

There was no statistical significance between OCD or SAD and any of the other predictors, including age, gender, marital status, socioeconomic status, or the clinical subtype of MS, which was in concordance with other similar studies except that in Foroughipour et al., where women were more likely to get OCD [11]. One study suggested a correlation between OCD prevalence and higher educational level among people with MS [26]. We did not find any relationship between educational level and OCD.

The study's cross-sectional design limits establishing causal relationships. Other study limitations may include the use of self-administered questionnaires that might have led to over or under-reporting, which could not be ascertained. The study participants mostly had mild to moderate disability based on EDSS scores; in addition, all participants were recruited from a tertiary center.

Therefore, the results may not be generalizable for all MS populations. Our study assessed the psychiatric conditions based on self-reported questionnaires without formal psychiatric evaluation. A study with formal psychiatric assessment may address the limitation of self-administered questionnaires. Finally, it is essential to investigate other associated mental disorders and their burden on patients living with multiple sclerosis to remove any confounding bias.

\section{Conclusions}

We found social anxiety and obsessive-compulsive disorder to be common among pwMS. There was an association between impaired walking and more prominent social anxiety symptoms. If SAD was present, the pwMS were more likely to have OCD, suggesting multiple mental comorbidities. These findings assert the need for psychiatric screening for persons with MS, as it is important to detect mental illnesses early for their large impact on pwMS lives and outcomes.

\section{Additional Information}

\section{Disclosures}

Human subjects: Consent was obtained or waived by all participants in this study. King Abdullah International Medical Research Center issued approval SP17-124R. Animal subjects: All authors have confirmed that this study did not involve animal subjects or tissue. Conflicts of interest: In compliance with the ICMJE uniform disclosure form, all authors declare the following: Payment/services info: All authors have declared that no financial support was received from any organization for the submitted work. Financial relationships: All authors have declared that they have no financial relationships at present or within the previous three years with any organizations that might have an interest in the submitted work. Other relationships: All authors have declared that there are no other relationships or activities that could appear to have influenced the submitted work.

\section{References}

1. Kamińska J, Koper OM, Piechal K, Kemona H: Multiple sclerosis-etiology and diagnostic potential . Postepy Hig Med Dosw. 2017, 30:551-563. 10.5604/01.3001.0010.3836

2. Goris A, Dubois B, Hilven K: Role of genetic factors in pathophysiology of multiple sclerosis . Multiple Sclerosis: A Mechanistic View. Minagar A (ed): Academic Press, Cambridge, Massachusetts; 2016. 153-180. 10.1016/B978-0-12-800763-1.00008-7

3. Dutta R, Trapp BD: Relapsing and progressive forms of multiple sclerosis. Insights from pathology . Curr Opin Neurol. 2014, 27:271-278. 10.1097/WCO.0000000000000094

4. World Health Organization. Atlas. Multiple sclerosis resources in the world 2008 . (2008). Accessed: November 15, 2019: https://apps.who.int/iris/bitstream/handle/10665/43968/9789241563758_eng.pdf.

5. Yamout BI, Assaad W, Tamim H, Mrabet S, Goueider R: Epidemiology and phenotypes of multiple sclerosis in the Middle East North Africa (MENA) region. Mult Scler J Exp Transl Clin. 2020, 6:2055217319841881. $10.1177 / 2055217319841881$

6. AlJumah M, Bunyan R, Al Otaibi H, et al.: Rising prevalence of multiple sclerosis in Saudi Arabia, a descriptive study. BMC Neurol. 2020, 20:49. 10.1186/s12883-020-1629-3 
7. Daif AK, Alrajeh S, Awada A, Al Bunyan M, Ogunniyi A, Abdul Jabar M, Al Tahan AR: Pattern of presentation of multiple sclerosis in Saudi Arabia: analysis based on clinical and paraclinical features. Eur Neurol. 1998, 93:182-186. 10.1159/000007931

8. Marrie RA, Reingold S, Cohen J, et al.: The incidence and prevalence of psychiatric disorders in multiple sclerosis: a systematic review. Mult Scler. 2015, 21:305-317. 10.1177/1352458514564487

9. Alamri Y, Al-Busaidi I S: Multiple sclerosis in Saudi Arabia: Anxiety, depression and sociability . Psychiatry Res. 2016, 238:224. 10.1016/j.psychres.2016.02.009.

10. Poder K, Ghatavi K, Fisk JD, et al.: Social anxiety in a multiple sclerosis clinic population . Mult Scler. 2009, 15:393-398. 10.1177/1352458508099143.

11. Foroughipour M, Behdani F, Hebrani P, Marvast MN, Esmatinia F, Akhavanrezayat A: Frequency of obsessive-compulsive disorder in patients with multiple sclerosis: A cross-sectional study. J Res Med Sci. 2012, 17:248-253.

12. Martin P: The epidemiology of anxiety disorders: a review . Dialogues Clin Neurosci. 2003, 5:281-298. 10.31887/DCNS.2003.5.3/pmartin

13. Zaudig M: Obsessive-compulsive disorder-clinical picture, diagnosis, and therapy [Article in English/German]. Z Psychosom Med Psychother. 2011, 57:3-50. 10.13109/zptm.2011.57.1.3

14. Aderka IM, Hofmann SG, Nickerson A, Hermesh H, Gilboa-Schechtman E, Marom S: Functional impairment in social anxiety disorder. J Anxiety Disord. 2012, 26:393-400. 10.1016/j.janxdis.2012.01.003

15. Gururaj GP, Bada Math S, Reddy JYC, Chandrashekar CR: Family burden, quality of life and disability in obsessive compulsive disorder: an Indian perspective. J Postgrad Med. 2008, 54:91-97. 10.4103/00223859.40773

16. Lublin FD: New multiple sclerosis phenotypic classification. Eur Neurol. 2014, 72:1-5. 10.1159/000367614

17. Connor KM, Davidson JR, Churchill LE, Sherwood A, Weisler RH, Foa E: Psychometric properties of the Social Phobia Inventory (SPIN): new self-rating scale. Br J Psychiatry. 2000, 176:379-386. 10.1192/bjp.176.4.379

18. Goodman WK, Price LH, Rasmussen SA, et al.: Yale-brown obsessive-compulsive scale (Y-BOCS) . Arch Gen Psychiatry. 1989, 46:1006-1011. 10.1001/archpsyc.1989.01810110048007

19. Beaton DE, Bombardier C, Guillemin F, Ferraz MB: Guidelines for the process of cross-cultural adaptation of self-report measures. Spine. 2000, 5:3186-3191. 10.1097/00007632-200012150-00014

20. Kurtzke JF: Rating neurologic impairment in multiple sclerosis. An expanded disability status scale (EDSS) . Neurology. 1983, 33:1444. 10.1212/wnl.33.11.1444

21. Beiske AG, Svensson E, Sandanger I, Czujko B, Pedersen ED, Aarseth JH, Myhr KM: Depression and anxiety amongst multiple sclerosis patients. Eur J Neurol. 2008, 15:239-245. 10.1111/j.1468-1331.2007.02041.x

22. Sarısoy G, Terzi M, Gümüş K, Pazvantoğlu O: Psychiatric symptoms in patients with multiple sclerosis . Gen Hosp Psychiatry. 2013, 35:134-140. 10.1016/j.genhosppsych.2012.10.011

23. Shabani A, Moghadam JA, Panaghi L, Seddigh A: Anxiety disorders in multiple sclerosis: significance of obsessive-compulsive disorder comorbidity. J Res Med Sci. 2007, 12:172-177. 10.1016/j.genhosppsych.2012.10.011

24. Mohamadirizi S: The survey of obsessive-compulsive disorder symptoms in patients with multiple sclerosis and its association with eating attitudes. J Mult Scler. 2016, 3: 1000179.

25. Baldwin DS, Brandish EK, Meron D: The overlap of obsessive-compulsive disorder and social phobia and its treatment. CNS Spectr. 2008, 13:47-53. 10.1017/s1092852900026936

26. Shabani A, Nikravesh S, Panaghi L: Obsessive-compulsive disorder: is it common in multiple sclerosis? . IJPCP. 2006, 12:209-215. 